\title{
Home cage behavior following chemical stimulation of the lateral hypothalamus
}

ROBERT J, BLANCHARD AND D. CAROLYN BLANCHARD

UNIVERSITY OF HAWAII

Cholinergic stimulation of the lateral hypothalamus of the rat was found to produce a reliable increment in drinking and home cage activity, and a reduction in sleeping. These results support the suggestion that the rat and the cat differ with respect to their reactions to lateral hypothalamic stimulation. Also, the hyperactivity accompanying cholinergic stimulation may be considered to represent a difference between cholinergically induced thirst and physiological thirst. Adrenergic stimulation failed to reliably modify the home cage behavior of the rat.

Several studies (Grossman, 1962a; Grossman, 1962b; Miller, 1965) have shown that the direct application of cholinergic substances to the lateral hypothalamus of the rat elicits drinking, while adrenergic stimulation of the same area produces eating. In the cat however, Hernández-Péon \& Chávez-Ibarra (1963) have reported that cholinergic stimulation of the lateral hypothalamus does not modify consummatory behavior but rather, induces sleep. This discrepancy has prompted Miller (1965) to suggest possible species differences in reactions to chemical stimulation.

Since previous investigations have not examined activity changes accompanying direct chemical stimulation of the rat hypothalamus, the possibility remains that changes similar to those seen in the cat may accompany cholinergic stimulation of the rat. Since cholinergic stimulation leads to a pronounced increase in drinking in the rat, activity changes from such stimulation might be regarded as secondary to this increase in motivation. However, Bolles (1965) has shown that prolonged water deprivation does not change the rat's home cage activity. Accordingly, any activity changes accompanying cholinergic stimulation of the rat hypothalamus could not be regarded as secondary to a normal increase in thirst.

\section{Method and Procedure}

The Ss were five male Holtzman derived rats from the colony maintained by the University of Hawaii Department of Psychology. They ranged in age from 156 to 160 days at the time of implantation. Chemitrodes were unilaterally implanted in the lateral hypothalamus ( $A=4.4 ; M L=1.8 ;$ subdural $=-7.0$ after König \& Klippel, 1963), using a procedure and materials similar to those of Grossman (1962a).

Five days after the operation, home cage rating was initiated. Each day, at approximately 4 p.m. each S's inner cannula was removed, cleaned, and replaced with either carbachol, norepinephrine or nothing (sham) in its tip a Five min. later, each S's behavior was rated as belonging to one of six categories: active, resting, sleeping, grooming, eating, or drinking. Ratings were continued at $30 \mathrm{sec}$. intervals, for $20 \mathrm{~min}$., which gave a total of 40 ratings per Sper session. The $E$ who rated these behaviors did not know which drug had been given. Each S received a total of three injections of carbachol, three of norepinephrine, and three sham injections. Conditions were presented according to a predetermined random schedule.

Following the experiment, all Ss were sacrificed and perfused with formaline. Fifty mu sections were taken of the area of implant, and stained according to the procedure described by Kluver \& Barrera (1951). Results

Location of placements. Four of the Ss were implanted in the lateral hypothalamus, from .4 to $.6 \mathrm{~mm}$ dorsolateral to the fornix. The fifth $S$ had an extremely enlarged lateral ventricle, on the side opposite its implanted chemitrode. This ventricular enlargement had compressed and distorted both the ipselateral and, to a certain extent, the contralateral, thalamus and hypothalamus. This $\mathrm{S}$ was therefore discarded, and all analyses are based on the remaining four animals.

Chemical stimulation. The only consistent change in consummatory activity with chemical stimulation was an increase in drinking for all $\mathrm{Ss}$, following carbachol administration. Two of the four Ss showed an increase in eating when given norepinephrine.

However, as may be seen in Fig. 1, carbachol produced a pronounced increase in activity, and decrease in

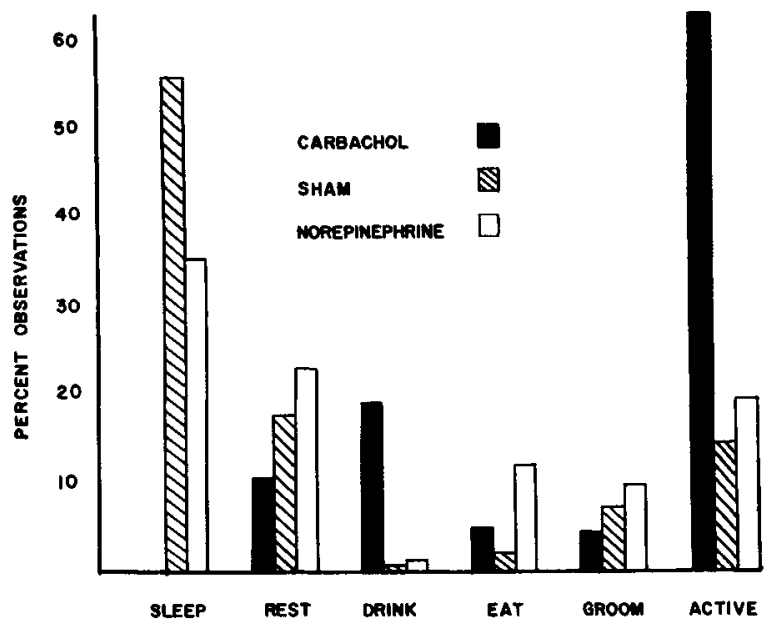

Fig. 1. Proportion of responses in each rating category as a function of type of hypothalamic stimulation. 
resting; following carbachol injection, sleeping was eliminated. To assess the reliability of these changes, ts for correlated measures were obtained: activity and grooming combined $(t=3.50 ; p<.05)$, sleeping and resting combined $(t=5.12 ; p<.02)$ and drinking $(t=5.55$; $p<.02)$ were reliably different, following carbachol injection, from sham injection values.

Although two Ss did show an increase in eating following norepinephrine, the group increase was not significant $(t=2.15 ; p>.05)$. No consistent or reliable changes in sleeping, resting, activity, or grooming followed norepinephrine injection.

\section{Discussion}

The results of the present study offer additional support for the suggestion that the rat and cat differ with respect to their reactions to cholinergic stimulation of the lateral hypothalamus. In addition to differences in consummatory behavior, rats apparently display hyperacitivity to such stimulation, in direct contrast to the sleep reactions displayed by the cat.

Since the appropriate blocking agents were not used, it was impossible to determine if this hyperactivity is specific to carbachol or cholinergic substances. Also, even though the hyperactivity obtained in the present study cannot be regarded as a secondary effect of normal thirst, it might be secondary to some other effect of cholinergic stimulation of the lateral hypothalamus. Miller (1965) has reported a decrease in body temperature with carbachol doses sufficient to elicit drinking; such temperature variations might well produce hyperactivity.

An additional possibility is that the novelty of the sudden thirst accompanying cholinergic stimulation, may produce hyperactivity, while normal thirst, devel- oping in a slow and gradual fashion, does not. Finally, cholinergic stimulation may directly affect pathways involved in both the regulation of behavioral arousal, and the regulation of thirst.

The failure of adrenergic stimulation to produce a reliable modification of home cage activity is not surprising. Only $50 \%$ of the Ss showed a consistent increase in eating following the administration of norepinephrine. Both Grossman (1962a) and Miller, Gottesman, \& Emery (1964) have reported similar proportions of Ss failing to eat following adrenergic stimulation of the lateral hypothalamus.

\section{References}

Bolles, Robert C. Effect of deprivation conditions upon the rat's home cage behavior. J. comp. physiol. Psychol., 1965, 60, 244248 .

Grossman, S. P. Direct adrenergic and cholinergic stimulation of hypothalamic mechanisms. Amer. J. Physiol., 1962a, 202, 872882.

Grossman, S. P. Effects of adrenergic and cholinergic blocking agents on hypothalamic mechanisms. Amer. J. Physiol., 1962b, 202, $1230-1236$.

Hernández-Péon, R., \& Chávez-Ibarra, G. Sleep induced by electrical or chemical stimulation of the forebrain. In R. HernándezPéon (Ed.), The physiological basis of mental activity. Amsterdam: Elsevier, 1963.

König, J. F. R., \& Klippel, R. A. The rat brain: A stereotaric atlas of the forebrain and lower parts of the brain. Baltimore: Williams and Wilkins Co., 1963.

Kluver, H., \& Barrera, E. A method for the combined staining of cells and fibers in the nervous system. J. neuropathol. exp. Neurol., 1953, 12, 400-406.

Miller, $\mathbf{N}$. Chemical coding of behavior in the brain. Science, 1965 , $148,328-338$.

Miller, N., Gottesman, K., \& Emery, N. Dose response to carbachol and norepinephrine in rat hypothalamus. Amer. J. Physiol., 1964, 206, 1384-1388. 